\title{
Hybrid solvation models for bulk, interface, and membrane: Reference interaction site methods coupled with density functional theory
}

\author{
S. Nishihara ${ }^{1}$ and M. Otani ${ }^{2}$ \\ ${ }^{1}$ ASTOM R\&D, 2-3-13 Minami, Wako-shi Saitama 351-0104, Japan \\ ${ }^{2}$ National Institute of Advanced Industrial Science and Technology (AIST), 1-1-1 Umezono, Tsukuba 305-8568, Japan
}

(Received 20 June 2017; revised manuscript received 31 August 2017; published 18 September 2017)

\begin{abstract}
We present two hybrid solvation models for the calculation of the solvation structure with model 1 in a confined nanospace in bulk materials and model 2 at solid/liquid interfaces where an electrode is in contact with an electrolyte and a membrane is immersed into a solution. The hybrid theory is based on the reference interaction site method (RISM) for the solvent region. The electronic structure of a bulk material, an electrode, and a membrane is treated by density functional theory with the plane-wave basis and pseudopotentials technique. For model 1, we use the three-dimensional RISM (3D-RISM) by imposing a 3D periodic boundary condition on the system. However, for model 2, we reformulate the RISM by means of a two-dimensional boundary condition parallel to the surface and an open boundary condition normal to the surface. Four benchmark calculations are performed for the formaldehyde-water system, water packed into a zeolite framework, a $\mathrm{NaCl}$ solution in contact with an $\mathrm{Al}$ electrode, and an $\mathrm{Al}$ thin film immersed in a $\mathrm{NaCl}$ solution with different concentrations. The calculations are shown to be efficient and stable. Because of the flexibility of the RISM theory, the models are considered to be applicable to a wide range of solid/liquid interfaces.
\end{abstract}

DOI: 10.1103/PhysRevB.96.115429

\section{INTRODUCTION}

The plane-wave (PW) basis set and pseudopotential (PP) technique often are used to calculate the electronic structures of condensed matter based on the Kohn-Sham density functional theory (DFT) [1-3]. Although the DFT-based PW and PP method (hereafter called the PW-PP method) has been successful for the solid state, some problems remain for the solution (mixture of ions/molecules in liquid). One of the most serious problems is to describe the thermal fluctuations of solvent molecules and ions. The direct approach to address this problem is molecular dynamics (MD) simulations [4,5], sometimes enhanced with the blue moon ensemble method [6]. However, MD requires a substantial computational cost. An alternate method with a lower computational cost is the implicit solvent theory (IST), which describes a solvent system as a continuum model. There are varieties of IST, and some of them have already been implemented into PW-PP: a method based on the polarizable continuum model (PCM) [7] or a classical DFT-based method [8].

In this paper, we focus on one of the ISTs, the reference interaction site method (RISM) [9]. The RISM already has succeeded in describing the solvation structures around various isolated or biological molecules using a classical force field [10,11] or Gaussian-based ab initio calculations [12,13]. Even though these calculations involve a low computational cost, they yield more thermodynamically relevant solvation structures for each solvent atom than other ISTs. If we could apply the RISM instead of MD to describe an electrolyte solution on an electrode under an electric bias, this will provide

Published by the American Physical Society under the terms of the Creative Commons Attribution 4.0 International license. Further distribution of this work must maintain attribution to the author(s) and the published article's title, journal citation, and DOI. various eminent advantages. Since the RISM can realize a noninteger treatment for ions contrary to the full-atomistic $\mathrm{MD}$, it may screen the electric field arising from the noninteger electron on the electrode, which is described by PW-PP to have a continuous density of states of electrons. The structure of the electric double layer (EDL) in the electrolyte solution near the electrode where different numbers (concentration) of positive and negative ions are located will be given by the RISM.

In the present paper, we combine the RISM and PWPP to develop two kinds of hybrid solvation model. One is based on a three-dimensional RISM (3D-RISM) [11,13] approach imposing the 3D periodic boundary condition (PBC) in which we can calculate the solvation structure in a confined nanospace in framework systems. This model is solved by a simple extension of the conventional 3D-RISM by coupling with PW-PP. The other is a model for solid/liquid interfaces. Contrary to the former model, an interface has a geometric restriction in the periodicity of the structure: We need to impose the two-dimensional (2D) PBC parallel to the surface and the open boundary condition (OBC) along the normal direction. This mixed boundary condition (MBC) is inherently important for the electrostatic interaction in the interface geometries and requires a novel strategy for the hybridization of the RISM and PW-PP. More specifically, one needs to make both the RISM and PW-PP compatible with the MBC. With regard to PW-PP, Otani and Sugino have developed a method called the effective screening medium (ESM) method to electrostatically incorporate the MBC into PW-PP [14].

In the context of the RISM calculation of the solvation structure at an interface, one possible route to tackle this problem is the 3D-RISM approach with a liquid and an atomistic slab interface [15] or a liquid and a uniform impenetrable wall interface [16]. Since a 3D Fourier transform (FT) was used in their approach, in this sense, they do not solve the RISM equations under the MBC. There has been a series of pioneering works for solving the RISM equation under the OBC. Woelki and co-workers developed the singlet RISM 
(SRISM) theory $[17,18]$ where the flat surface is assumed as a part of the spherical particle with an infinite radius. They elucidated an asymptotic behavior of the direct correlation function which satisfies the RISM equation under the OBC. Since the SRISM theory is a reduced form of the RISM along the surface lateral direction, it is difficult to consider a surface structure, such as a step/terrace structure, surface defects, and any other more realistic surface structures. In the present paper, to overcome these difficulties, we reformulate the 3D-RISM by imposing the MBC and combine this with PW-PP in a fully self-consistent manner. The newly developed hybrid solvation models are implemented into the first-principles software suite of QUANTUM ESPRESSO [19].

\section{THE 3D-RISM}

Kovalenko and Hirata developed the original concept of the 3D-RISM with a partially linearized closure equation $[11,15]$ to simulate the distributions of a "solvent", such as water and ions, around a "solute," such as a biomolecule. The interaction between the solute and the solvent is described as a classical force field modeled by the Lennard-Jones (LJ) potential and point charges (see Eq. (S6) of the Supplemental Material [20]). To improve the modeling of the solute molecule, an ab initio electronic structure calculation with a Gaussian basis set was applied to a solute molecule by Sato and co-workers [13]; this method is called the 3D-RISM self-consistent field (3DRISM-SCF). In this method, the Coulombic interaction from the point charges is replaced by the electrostatic potential from the $a b$ initio calculation (see Eq. (S20) of the Supplemental Material [20]). Here, we develop a variant of the 3D-RISMSCF, using PW-PP as a solute system. In this method, the correlation functions of the 3D-RISM are defined in a unit cell of the PW-PP calculation. Also, the PBC is imposed on both the 3D-RISM and the PW-PP.

Contrary to an isolated molecule as a solute located inside the RISM, in our hybrid solvation model, we treat a bulk (host) material, such as zeolite [21], clay minerals [22], or organic frameworks [23], as a solute molecule located inside the RISM. In this case, we cannot define a reference for the distribution function (the region where it becomes unity) because of the 3D PBC. The issue arises from the uncertainty of the origin of the electrostatic potential in the 3D PBC system. Here we impose an auxiliary condition called the canonical condition on the distribution function,

$$
\int d \mathbf{r} g_{\alpha}(\mathbf{r})=V_{\text {cell }},
$$

where $g_{\alpha}$ is the distribution function of the $\alpha$ th solvent atom and $V_{\text {cell }}$ is the volume of a unit cell. This condition fixes the total number of solvent atoms and remedies the uncertainty of the reference. For more details, see Sec. II G in the Supplemental Material [20].

First, we demonstrate the 3D-RISM for two different isolated solute molecules: water and formaldehyde. The solute was centered in a unit cell of $20 \times 20 \times 20 \AA^{3}$ and calculated by DFT where the exchange functional was Becke88 [24] and the correlation functional was Lee-Yang-Parr [25]. Ultrasoft pseudopotentials were used for every atom. The cutoff energies of the wave function and charge density were 25 and
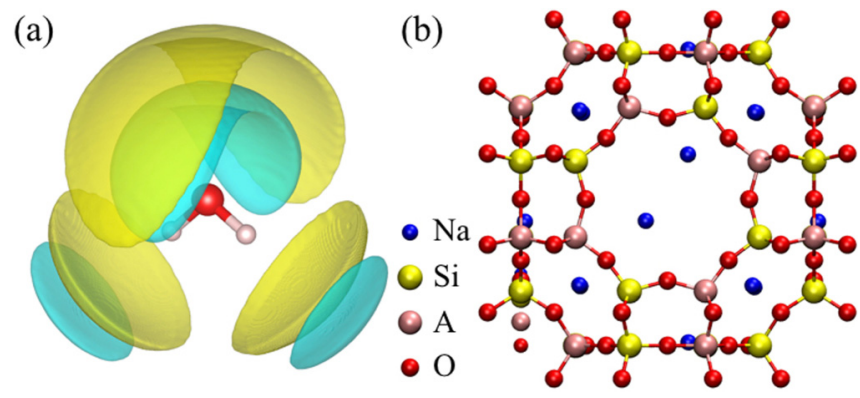

FIG. 1. (a) Isosurfaces of the solvent distribution functions around a solute water molecule. The yellow (light blue) isosurface refers to the oxygen (hydrogen) distribution in solvent water. The red and white spheres are oxygen and hydrogen atoms, respectively, of the solute. (b) The unit cell of the LTA zeolite including 12 sodium ions. An experimental lattice constant (12.28 $\AA$ ) is used [21]. The structure of the LTA framework is fixed, and the positions of the sodium ions are optimized.

225 Ry, respectively. Sampling in the Brillouin zone was only at the $\Gamma$ point. The solvent system was water with a density of $1 \mathrm{~g} / \mathrm{cm}^{3}$ and a temperature of $300 \mathrm{~K}$. The 3D-RISM was performed with the closure of the model of Kovalenko and Hirata (KH) [15]. The pair distribution functions obtained by the 3D-RISM with the closure of the KH model are known to well reproduce those obtained by molecular dynamic simulations (see, e.g., Ref. [26] and references therein). The fast Fourier transformation (FFT) technique was used to solve the integral equation of the solvent where the cutoff energy in the reciprocal space was $144 \mathrm{Ry}$. The simple point charge (SPC) model [27] was applied to water, and the optimized potentials for the liquid simulations all-atom force field [28] were applied to the LJ parameters of formaldehyde. Correlation functions of the 3D-RISM were converged with modified direct inversion of the iterative subspace [29]. Additionally, the electronic charge density and atomic geometry were optimized simultaneously. The calculated solvent water distribution around a solute water molecule, which was decomposed into the distributions of oxygen and hydrogen, is shown in Fig. 1(a). One can observe that the solvent hydrogen was coordinated with the solute oxygen atom and the solvent oxygen was coordinated with the solute hydrogen atoms creating hydrogen bonds. Table I shows our calculated hydration free energies as well as other calculated data and the experimental data. For a water molecule, the present paper yielded good agreement with the experimental value and the results of the 1D-RISMSCF [30], the 3D-RISM-SCF [30], and the MOZ-SCF [31]. For the hydration free energy of a formaldehyde molecule obtained by various simulations, there was a rather pronounced discrepancy with the experimental data. Although the origin of the discrepancy has been discussed previously [31], this was beyond the scope of the present paper.

To illustrate water packing into a framework system, we calculated a zeolite of Linde type A (LTA) [21], whose unit cell is shown in Fig. 1(b). The LTA framework and sodium ions were calculated by PW-PP where the exchange-correlation functional was the Perdew-Burke-Ernzerhof (PBE) [34]. Norm-conserving pseudopotentials were used for silicon and aluminum atoms, and ultrasoft pseudopotentials were used for sodium and oxygen atoms. Sampling in the Brillouin zone was 
TABLE I. Calculated and experimental hydration free energies of water and formaldehyde molecules. In the present paper, the hydration free energy is calculated by the KH model. The data of Gaussianbased $a b$ initio calculations with the PCM, the 1D-RISM-SCF, the 3D-RISM-SCF, and the MOZ-SCF approaches are shown.

\begin{tabular}{llr}
\hline \hline & \multicolumn{2}{l}{ Hydration free energy/kcal mol } \\
\cline { 2 - 3 } & $\mathrm{H}_{2} \mathrm{O}$ & $\mathrm{HCHO}$ \\
\hline PCM $^{\mathrm{a}}$ & -1.4 & 2.7 \\
1D-RISM-SCF $^{\mathrm{b}}$ & -5.1 & 4.1 \\
3D-RISM-SCF $^{\mathrm{b}}$ & -5.6 & 1.9 \\
MOZ-SCF $^{\mathrm{a}}$ & -4.2 & 1.3 \\
The present paper & -4.2 & 2.3 \\
Experiment & $-6.3^{\mathrm{c}}$ & $-1.7^{\mathrm{d}}$ \\
\hline \hline
\end{tabular}

${ }^{\text {a Reference [31]. }}$

${ }^{b}$ Reference [30].

${ }^{\mathrm{c}}$ Reference [32].

${ }^{\mathrm{d}}$ Reference [33].

performed using the $2 \times 2 \times 2 k$ point of Monkhorst-Pack [35]. Water packed inside of the framework was treated as a continuum distribution of the 3D-RISM with the closure of the KH model. Cutoff energies were 25, 225, and $169 \mathrm{Ry}$ for the electronic wave function, charge density, and solvent correlation function, respectively. LJ parameters of the LTA framework were the Clay-FF force field [22], but those of the sodium ions were taken from Ref. [32]. Water was treated by the SPC model, and its temperature was $300 \mathrm{~K}$. Because we have the canonical condition for water [Eq. (1)], the calculation was performed with various numbers of water molecules (Fig. 2). The total energy became most stable when 25 water molecules were packed. This result is reasonable as 27 water molecules were observed experimentally [21].

\section{THE LAUE-RISM}

The 3D PBC defined on the 3D-RISM assists us in applying the FFT technique. Then, the integral equation of the RISM

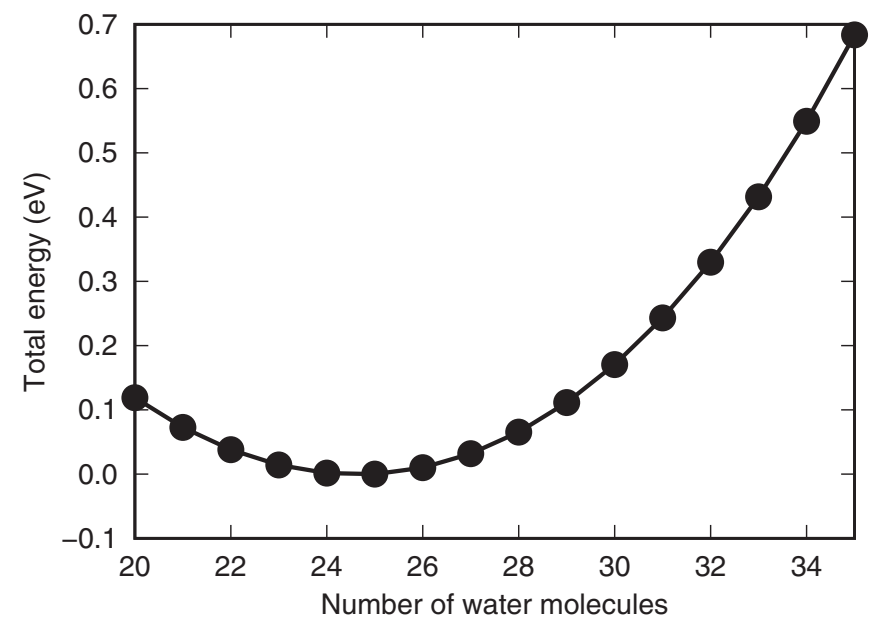

FIG. 2. Calculated total energies of the LTA zeolite for various numbers of water molecules. The total energy is the sum of the DFT energy and solvation free energy calculated by KH's closure [15]. can be solved very easily. However, the MBC does not have $3 \mathrm{D}$ periodicity, and we cannot apply the conventional 3D-FFT technique. Hence, we rewrite the RISM equation using the Laue representation, which is compatible with the MBC,

$$
h_{\gamma}\left(\mathbf{g}_{\|}, z\right)=\sum_{\alpha} \int_{-\infty}^{\infty} d z^{\prime} c_{\alpha}\left(\mathbf{g}_{\|}, z^{\prime}\right) \chi_{\alpha \gamma}\left(\mathbf{g}_{\|}, z^{\prime}-z\right),
$$

where $h_{\gamma}$ is the total correlation function of the $\gamma$ th solvent atom, $c_{\alpha}$ is the direct correlation of the $\alpha$ th solvent atom, $\chi_{\alpha \gamma}$ is the susceptibility between the $\alpha$ th and the $\gamma$ th solvent atoms, and $\mathbf{g}_{\|}$is a wave vector parallel to the surface. Hereafter, we call this method the Laue-RISM. Equation (2) requires more computational cost than that of the conventional 3D-RISM and becomes a bottleneck for the Laue-RISM calculation. The order of computation is $O\left(N^{4 / 3}\right)$, where $N$ is the number of grids in the box of the FFT. However, this computational order is much smaller than that of the DFT calculation $\left[O\left(N^{2-3}\right)\right]$. Especially for large systems, the computational time of the Laue-RISM may be negligible.

Although introducing Eq. (2) allows us to simulate the exact solvent distributions around an isolated slab, the conventional PW-PP calculation still has the PBC, and slabs are repeated along the $z$ direction. To combine with the Laue-RISM, the PBC along this direction needs to be removed from PW-PP. This requirement for PW-PP is achieved efficiently by the ESM method. The ESM method solves the Poisson equation using the Laue represented Green's function to yield a nonrepeated electrostatic potential from an isolated slab. Although the ESM method can supply various boundary conditions, in the present paper, we applied the OBC in the ESM method which consists of a vacuum/slab/vacuum configuration [14]. There were two vacuum regions on the left and the right sides of the slab. Domains of the solvent system were put on the left-hand side and/or the right-hand side. Combining the ESM method with the Laue-RISM, the configuration of the systems became vacuum/slab/solvent [see Fig. 3(a)] and solvent/slab/solvent [see Fig. 4(a)]. We called this method the ESM-RISM. The free energy $A[15]$ of the ESM-RISM is defined as

$$
A=E_{\mathrm{DFT}}+\Delta \mu_{\mathrm{solv}},
$$

where $E_{\mathrm{DFT}}$ is the total energy from the DFT calculation and $\Delta \mu_{\text {solv }}$ is the solvation free energy calculated by the RISM. If the slab is neutral, $A, E_{\mathrm{DFT}}$, and $\Delta \mu_{\text {solv }}$ are defined uniquely. However, $E_{\mathrm{DFT}}$ of the charged slab cannot be defined because the electrostatic energy calculated by the ESM with the OBC diverges. However, $\Delta \mu_{\text {solv }}$ also diverges because the induced net charge leads to a diverged behavior in the electrostatic energy of the Laue-RISM. Although $E_{\mathrm{DFT}}$ and $\Delta \mu_{\text {solv }}$ diverge, both of the divergence terms cancel out each other to yield a finite $A$ (details are in the Supplemental Material [20]). Thus even if a slab is charged, the ESM-RISM is always able to define the total energy of a solvated system. This fact means that ions in a solvent system completely screen out the excess charge of the slab and eventually the whole system becomes neutral. This spontaneous charge neutrality condition is the most important advantage of the ESM-RISM.

The following two points are worth mentioning. (1) In our hybridization method since the solvation potential (Eqs. (S71) and (S72) in the Supplemental Material [20]) 


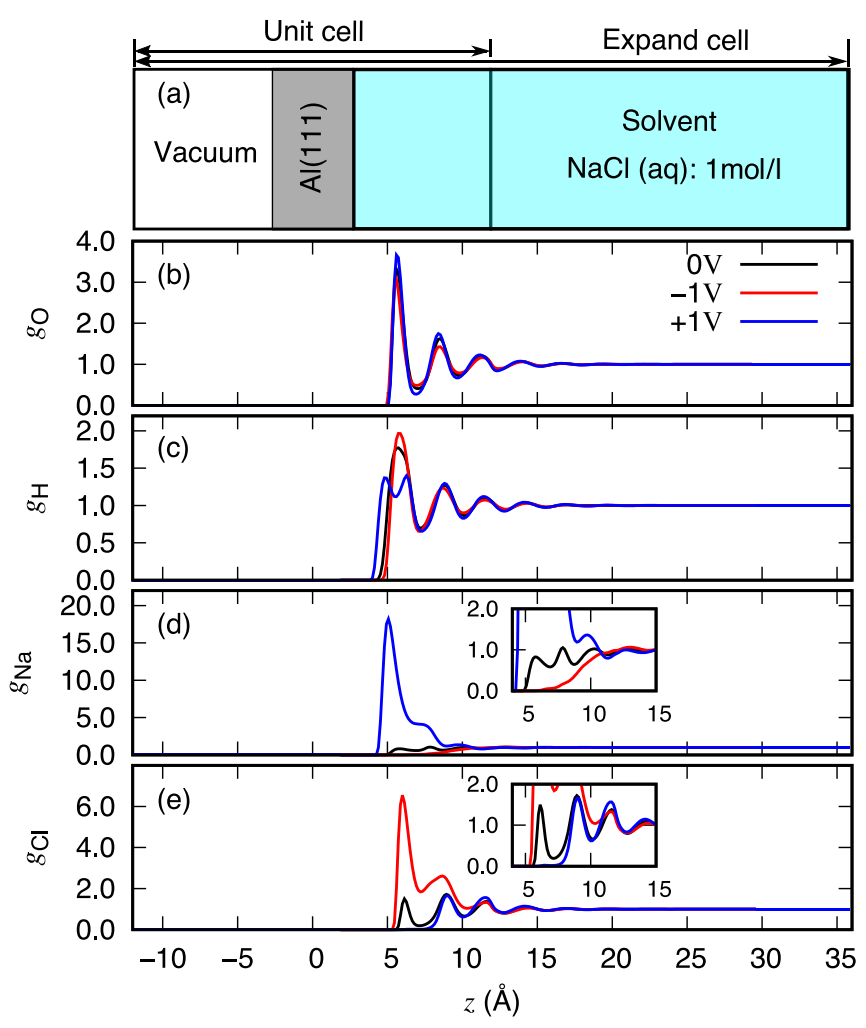

FIG. 3. (a) Geometric configuration of the solid/liquid interface (vacuum/slab/solvent model). Normalized distribution function for (b) $\mathrm{O}$, (c) $\mathrm{H}$, (d) Na, and (e) $\mathrm{Cl}$ with different bias potentials. The Fermi energies of the systems are $-5.3,-4.3$, and $-3.3 \mathrm{eV}$ for -1.0 , 0.0 , and $+1.0 \mathrm{~V}$ vs pzc, respectively.

acts as a confining potential, the electrons in the PW-PP region do not spill out into the RISM region. This means that there is no need to introduce a barrier potential to prevent electron leakage from the PW-PP region. (2) The asymptotic behavior of the direct correlation function is critical to ensure the charge neutrality condition as pointed out by Woelki and co-authors [18]. We also checked the sum rule [17] for the direct correlation function and confirmed that the rule was satisfied in our calculation (see the Supplemental Material [20]).

PW-PP calculation was performed for a slab of aluminum (111) where the PBE exchange-correlation functional, normconserving pseudopotential, and a $4 \times 4 \times 1 k$-point mesh of Monkhorst-Pack sampling were used. The optimized lattice constant of the aluminum crystal was $4.04 \AA$. The slab was constructed with a $2 \times 2$ supercell and three atomic layers, including 12 atoms. The solvent system was an aqueous (aq) solution of sodium chloride with a concentration of $1.0 \mathrm{~mol} / 1$ and a temperature of $300 \mathrm{~K}$ where the force field of water was a SPC and those of the ions were treated with the model by Smith et al. [36]. Additionally, the Laue-RISM was performed with a closure of the $\mathrm{KH}$ model. The cutoff energies were 25 and $144 \mathrm{Ry}$ for the electronic wave function and solvent correlation function, respectively. The universal force field [37] was used for the LJ parameters of the solute aluminum atoms. The length of the unit cell along the $z$ axis was $24 \AA$ for the PW-PP calculation and was expanded to $48 \AA$ for the Laue-RISM

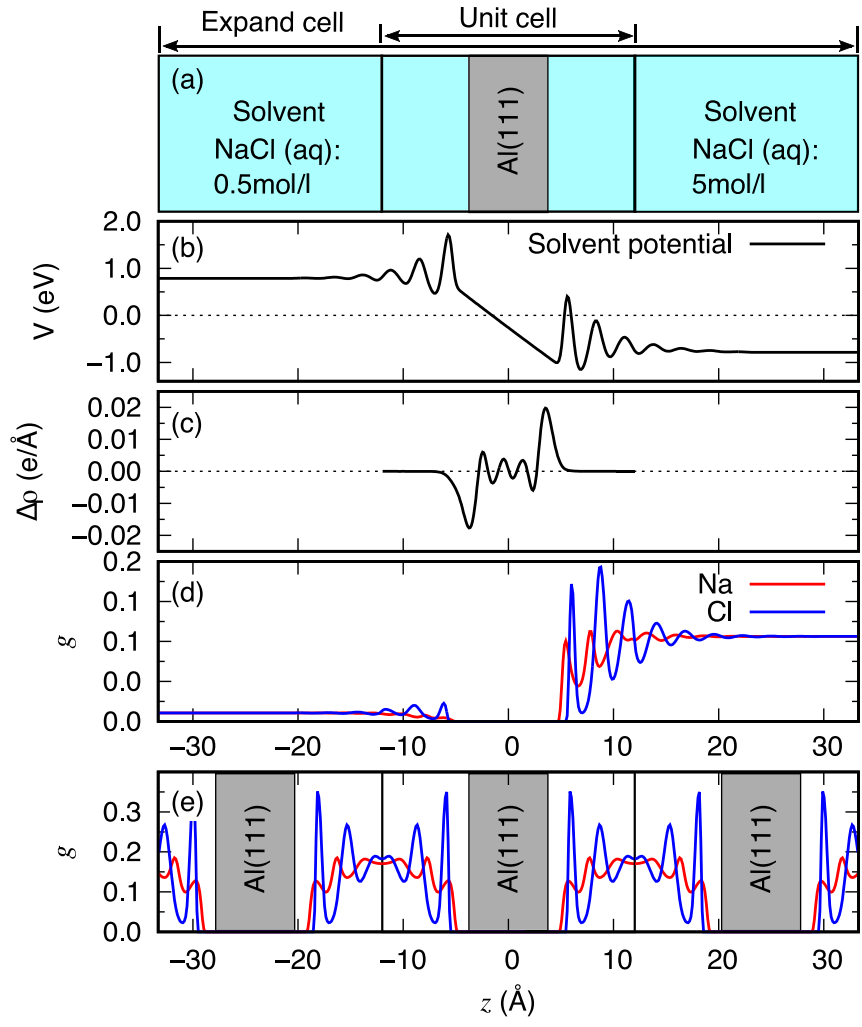

FIG. 4. (a) Geometric configuration of the solid/liquid interface (solvent/slab/solvent model). (b) Electrostatic potential for the solvent. (c) The difference charge density from an isolated $\mathrm{Al}$ thin film. (d) Distribution function for $\mathrm{Na}$ and $\mathrm{Cl}$. (e) Distribution function for $\mathrm{Na}$ and $\mathrm{Cl}$ with a 3D-RISM calculation (see the text).

calculation [Fig. 3(a)]. Calculated solvent distributions are shown in Figs. 3(b)-3(e), which were evaluated as densities integrated on the $x y$ plane. Water molecules were coordinated with the aluminum surface by hydrogen atoms in the first layer. The amplitudes of the solvent diminish to zero, and the densities eventually become a constant value.

We also performed two other calculations, applying the bias potential $+1.0 \mathrm{~V}$ vs pzc and $-1.0 \mathrm{~V}$ vs pzc to the aluminum slab. Here, pzc refers to the potential of zero charge, which is defined by the Fermi energy of the neutral slab calculation. To apply the bias, we use the constant bias potential scheme developed by Bonnet and co-workers [38]. The distribution of the solvent atoms and ions depending on the bias also are shown in Figs. 3(b)-3(e). When $-1.0 \mathrm{~V}$ vs pzc was applied (red curves), $\mathrm{Na}^{+}$decreased, and $\mathrm{Cl}^{-}$increased near the surface. Oppositely, applying $+1.0 \mathrm{~V}$ vs pzc (blue curves) caused an increase in $\mathrm{Na}^{+}$and a decrease in $\mathrm{Cl}^{-}$. The total charges of the solvent (slab) system were $-0.2 e(+0.2 e)$ for $-1.0 \mathrm{~V}$ vs pzc and $+0.5 e(-0.5 e)$ for $+1.0 \mathrm{~V}$ vs pzc, then the total system was always neutral. This means that we can obtain the solvent distribution under the grand canonical condition for a solvent system and describe the EDL on the charged surface. This is an impressive advantage of the ESM-RISM. Unlike the continuum model, such as the PCM [39], we can directly obtain the detailed solvation structure of the EDL and discuss the bias dependence of the EDL structure at a realistic electrode/electrolyte interface. Although a simple comparison 
with previous RISM calculations was not relevant here because of the difference in the model and parameters, the qualitative behavior of the peaks for density profiles were similar to those charge/uncharged wall results [16-18].

As an example of a membrane, we performed a solvent/slab/solvent calculation with a neutral aluminum slab. We included aqueous solutions of $\mathrm{NaCl}$ on both sides where these solutions had different concentrations. The $\mathrm{NaCl}$ (aq) solution on the right side had a concentration of $5.0 \mathrm{~mol} / \mathrm{l}$, and that on the left side had a concentration of $0.5 \mathrm{~mol} / \mathrm{l}$. Figure 4(a) shows a geometrical configuration of the model. We used the same unit cell $(24 \AA)$ as the previous model, and it was expanded to $72 \AA$ for the Laue-RISM calculation. As shown in Fig. 4(b), the imbalance of the ionic concentration results in the different electrostatic potentials on both sides. Consequently, there was a concerted charge redistribution: The electron on the left surface was depleted, and that on the right surface was accumulated [Fig. 4(c)]. Conversely the surface charge was screened by an increased presence of the counterions [Fig. 4(d)].

Finally, to compare the ESM-RISM and the 3D-RISM, we also conducted 3D-RISM calculations using the same unit cell. For the concentration of the aqueous solution of $\mathrm{NaCl}$, we only prepared a $5.0 \mathrm{~mol} / 1$ solution because the PBC does not allow for including different concentration solutions on both sides of the slab in the 3D-RISM. As shown in Fig. 4(e), the distribution functions near the surface region (the first and second peaks) showed different profiles compared with those of the ESM-RISM. This means that there was a spurious interaction that affects the shape of the distribution function. To avoid this periodic image effect, one needs to expand the unit cell along the $z$ axis so that the spurious interaction becomes sufficiently small. Accordingly, the expansion of the unit cell for PW-PP substantially increases the calculation cost.

The results indicate that our hybrid solvation ESM-RISM model allows us to calculate a thin film immersed into a solvent with moderate calculation cost. Owing to the flexibility of the RISM, we can change the concentration of the ion and $p \mathrm{H}$ in both solvent regions independently. Additionally, we can perform calculations in a mixed solvent system.

\section{SUMMARY AND DISCUSSION}

We have modified the 3D-RISM to introduce the canonical condition [Eq. (1)]. This modification allows us to calculate not only an isolated molecular system, but also a framework system. In the present paper, we also have developed a different formulation of the RISM: the Laue-RISM. It is illustrated that a vacuum/slab/solvent calculation can be applied to a charged slab system, which adjoins an aqueous solution, such as an electrode/electrolyte interface. The calculation results for the solvent/slab/solvent imply that this model allows us to simulate an ion-exchange or biological membranes.

For the vacuum/slab/solvent model, one of the most important applications is the electrochemical reaction at the electrode/electrolyte interface. However, the model shown in Fig. 3(a) cannot directly simulate the electrochemical reactions, such as a water dissociation on the electrode and corrosion of the electrode because the RISM cannot accommodate such a reaction. We would like to emphasize here that, by including a water molecule described by PW-PP as a reacting species at the interface region, we can calculate the electrochemical reactions within the ESM-RISM framework. This flexibility of the modeling is a significant advantage of our method.

Besides the flexibility of the Laue-RISM, there are a few more advantages of the method: First is the calculation cost, second is the possibility to define the bias potential, and third is the exact treatment of the image charge contribution in the interaction between the slab electrode and the solvent atoms. In the ESM-RISM, we can use the minimum unit cell for the PW-PP slab calculation [see the unit cells in Figs. 3(a) and 4(a)]. This means that we do not need to increase the number of PWs with respect to the conventional PW-PP slab calculation. The second advantage is that, since we impose the OBC along the $z$ direction, we can define the electrostatic potential at the uniform solvent region (far from the surface) as the origin of the electrostatic potential. Thus, we have a rigorous definition of the electrode potential from the Fermi energy of the system. The last advantage is that, since we apply the Green's function in the electrostatic interaction between the slab and the solvent atoms, the spurious interaction arising from the mirror image inside the slab is included automatically in the ESM-RISM calculation. Generally, it is difficult to include such an interaction in classical molecular dynamics simulations and conventional RISM calculations. These advantages will be important advances in the electrochemical interface simulations.

\section{ACKNOWLEDGMENTS}

This work was partially supported by the R\&D Initiative for scientific innovation of new generation batteries 2 Project (RISING 2), the ultra-high-throughput design and prototyping technology for ultra-advanced materials development Project (Project No. P16010) administrated by the New Energy and Industrial Technology Department Organization (NEDO), by the MEXT Projects of "Priority Issue on Post-K computer" (development of new fundamental technologies for highefficiency energy creation, conversion/storage, and use), and the Elements Strategy Initiative for Catalysts and Batteries (ESICB).
[1] W. Kohn and L. J. Sham, Phys. Rev. 140, A1133 (1965).

[2] A. M. Rappe, K. M. Rabe, E. Kaxiras, and J. D. Joannopoulos, Phys. Rev. B 41, 1227 (1990).

[3] D. Vanderbilt, Phys. Rev. B 41, 7892 (1990).
[4] T. Todorova, A. P. Seitsonen, J. Jutter, I.-F. W. Kuo, and C. J. Mundy, J. Phys. Chem. B 110, 3685 (2006).

[5] D. Asthagiri, L. R. Pratt, and J. D. Kress, Phys. Rev. E 68, 041505 (2003). 
[6] E. A. Carter, G. Ciccotti, J. T. Hynes, and R. Kapral, Chem. Phys. Lett. 156, 472 (1989).

[7] O. Andreussi, I. Dabo, and N. Marzari, J. Chem. Phys. 136, 064102 (2012).

[8] S. A. Petrosyan, J. F. Briere, D. Roundy, and T. A. Arias, Phys. Rev. B 75, 205105 (2007).

[9] J. P. Hansen and I. R. McDonald, Theory of Simple Liquids (Academic, London, 1976).

[10] F. Hirata and P. J. Rossky, Chem. Phys. Lett. 83, 329 (1981).

[11] A. Kovalenko and F. Hirata, Chem. Phys. Lett. 290, 237 (1998).

[12] S. Ten-no, F. Hirata, and S. Kato, J. Chem. Phys. 100, 7443 (1994).

[13] H. Sato, A. Kovalenko, and F. Hirata, J. Chem. Phys. 112, 9463 (2000).

[14] M. Otani and O. Sugino, Phys. Rev. B 73, 115407 (2006).

[15] A. Kovalenko and F. Hirata, J. Chem. Phys. 110, 10095 (1999).

[16] J. J. Howard, J. S. Perkyns, and B. M. Pettitt, J. Phys. Chem. B 114, 6074 (2010).

[17] S. Woelki, H.-H. Kohler, and H. Krienke, J. Phys. Chem. B, 111, 13386 (2007).

[18] S. Woelki, H.-H. Kohler, and H. Krienke, J. Phys. Chem. B, 112, 3365 (2008).

[19] P. Giannozzi et al., J. Phys.: Condens. Matter 21, 395502 (2009).

[20] See Supplemental Material at http://link.aps.org/supplemental/ 10.1103/PhysRevB.96.115429 for details of the theory.

[21] P. A. Howell, Acta. Crystallogr. 13, 737 (1960).

[22] R. T. Cygan, J.-J. Liang, and A. G. Kalinichev, J. Phys. Chem. B 108, 1255 (2004).
[23] H. J. Zhou and S. Kitagawa, Chem. Soc. Rev. 43, 5415 (2014).

[24] A. D. Becke, Phys. Rev. A 38, 3098 (1988).

[25] C. Lee, W. Yang, and R. G. Parr, Phys. Rev. B 37, 785 (1988).

[26] M. C. Stumpe, N. Blinov, D. Wishart, A. Kovalenko, and V. S. Pande, J. Phys. Chem. B 115, 319 (2011).

[27] M. Matsugami, N. Yoshida, and F. Hirata, J. Chem. Phys. 140, 104511 (2014); the LJ parameters of hydrogen are modified, see Ref. [15].

[28] G. A. Kaminski, R. A. Friesner, J. Tirado-Rives, and W. L. Jorgensen, J. Phys. Chem. B 105, 6474 (2001).

[29] A. Kovalenko, S. Ten-no, and F. Hirata, J. Comput. Chem. 20, 928 (1999).

[30] N. Minezawa and S. Kato, J. Chem. Phys. 126, 054511 (2007).

[31] N. Yoshida and S. Kato, J. Chem. Phys. 113, 4974 (2000).

[32] A. Ben-Naim and Y. Marcus, J. Chem. Phys. 81, 2016 (1984).

[33] C. Amovilli and B. Mennucci, J. Phys. Chem. B 101, 1051 (1997).

[34] J. P. Perdew, K. Burke, and M. Ernzerhof, Phys. Rev. Lett. 77, 3865 (1996).

[35] H. J. Monkhorst and J. D. Pack, Phys. Rev. B 13, 5188 (1976).

[36] D. E. Smith and L. X. Dang, J. Chem. Phys. 100, 3757 (1994).

[37] A. K. Rappe, C. J. Casewit, K. S. Colwell, W. A. Goddard III, and W. M. Skiff, J. Am. Chem. Soc. 114, 10024 (1992).

[38] N. Bonnet, T. Morishita, O. Sugino, and M. Otani, Phys. Rev. Lett. 109, 266101 (2012).

[39] S. Miertuš, E. Scrocco, and J. Tomasi, Chem. Phys. 55, 117 (1981). 